Title:

\title{
A New Adaptive Framework for Unbiased Orientation Estimation in Textured Images.
}

\section{Author names and affiliations:}

\author{
Franck Le Pouliquen ${ }^{1}$, Jean-Pierre Da Costa ${ }^{1,2}$, Christian Germain ${ }^{1,2}$, Pierre Baylou ${ }^{1}$ \\ ${ }^{1}$ LAPS - Equipe Signal et Image - UMR CNRS n॰5131- GdR ISIS \\ ENSEIRB, BP 99, 33402 Talence cedex - France \\ ${ }^{2}$ LAPS - Equipe Signal et Image - UMR CNRS n०5131- GdR ISIS \\ ENITA de Bordeaux, BP 201, 33175 Gradignan cedex - France \\ jp-dacosta@enitab.fr c-germain@enitab.fr baylou@,tsi.u-bordeaux.fr
}

\section{Corresponding author:}

\author{
Christian Germain \\ LAPS - Equipe Signal et Image \\ Université Bordeaux 1 \\ 351, Cours de la Libération, F33405 Talence cedex - France \\ Tel +33540006142 Fax +33 540008406 \\ c-germain@enitab.fr
}

\begin{abstract}
This paper focuses on directional texture analysis. We propose a new approach for orientation estimation. This approach hinges on two classes of convolution masks, i.e. the gradient and the valleyness operators. We provide a framework for their optimization regarding bias reduction and noise robustness.

As the gradient and the valleyness operators are complementary, we propose a combination named GV-JOE. This combination consists in using the gradient on inflexion pixels, the valleyness on crests and valleys, and a linear mixture of both of them elsewhere. We implement an adaptive selection of the size of our operators, in order to take into account the variations of the texture scale in the image.

We exercise our approach both on synthetic and natural textures. These experiments show that, when used separately, both classes of operators are more accurate than classical derivative approaches. In noisy cases, the GV-JOE implementation improves the robustness of our operators without affecting their accuracy. Moreover, compared to well known orientation estimators, it gives the best estimates in the most difficult cases i.e. for high frequency textures and low SNR.
\end{abstract}

\section{Keywords:}

Orientation estimation, directional texture, convolution masks, unbiased estimation, texture analysis. 


\section{Introduction}

Directionality has been identified as one of the basic features used for image perception. For instance, orientation is one of the characteristics of textons used in pre-attentive vision [1]. It was also proved to be one of the three fundamental properties influencing texture recognition along with complexity and periodicity [2]. These textural properties can occur either simultaneously or separately.

This paper focuses on directional texture analysis i.e. on textures that show a spatial arrangement of oriented patterns. Examples of such textures can be found in various domains like fingerprint identification [3][4], oil prospecting or material structure characterization [5][6] (see Figure 1).

The characterization, the classification or the segmentation of directional textures must take into account the prop-

Fig. 1 erty of directionality. Many authors have addressed such tasks using the orientations of the patterns (e.g. [3][7]). For this purpose, the computation of an orientation map is an essential step.

In this paper, we assume that orientation estimation must rely on some basic rules.

First, orientation estimation should never be dissociated from the scale at which this orientation is considered. Indeed, the perception of a texture and its anisotropy depend on the scale at which it is observed [8][6]. For example, if the texture of Figure 2 is observed through window \#1, the perceived orientation is $45^{\circ}$. But at a larger observation scale (window \#2), the same texture shows a dominant orientation of $80^{\circ}$. In fact, the observation scale notion is a generalization of micro-texture and macro-texture concepts reported in previous works [9].

Fig. 2 A second important feature concerns the measure of the confidence we have on the orientation estimation. The estimation of the orientation relies on the assumption that the texture is locally oriented which is not always true. For instance, the orientation may not be defined on noisy regions or on boundaries of regions with different orientations. In such cases the use of a confidence or coherence index [10][11] is necessary.

Thirdly, some applications require a measure of orientation as accurate as possible (e.g. [5]). This implies that the operator used for orientation computation should be chosen carefully in order to take the noise sensitivity and the precision of the estimation into account.

Several approaches to the issue of orientation estimation have been proposed for the last three decades. The first attempts occurred in the context of edge detection. Indeed, the boundary between two regions with different magnitudes is characterized by a maximum of the gradient magnitude. The orientation of the gradient is the orientation of the boundary. Lyvers and Mitchell [12] provide with a survey of gradient operators in the framework of boundary orientation measurement. They study the bias and the noise sensitivity of several operators. Two kinds of implementations can be found in literature. They are based on either FIR or IIR filters.

The most popular FIR filters were proposed by Sobel or Prewitt [12]. The small size of these operators involves a strong sensitivity to noise. However, they are frequently used in cases where the orientation shows great variations at a local scale [13][7][6]. Some authors have derived variant versions of $3 \times 3$ masks optimized for orientation estimation [12][14].

The 1D approach followed by Canny [15] consists in finding an optimal FIR filter in the context of edge detection. This filter is designed according to three criteria. A detection criterion expresses that important edges should not be missed. A localization criterion minimizes the distance between the actual and located position of the edge. The last criterion reduces the likelihood of spurious responses to a single edge. The complexity of the solution led the author to choose an approximation of the solution based on the first derivative of a Gaussian function.

Following the same approach, Deriche [16][17] proposed a solution in the form of an IIR filter. The horizontal and vertical components of Deriche's gradient combine a smoothing filter and a derivative filter. The scale of the operator is tuned by an appropriate parameter chosen according to the size of the edges. Moreover, Deriche gives a recursive implementation of the filters.

Some approaches implementing IIR filters have been proposed. They are based on the derivative of a Gaussian [10] [18][11]. Other are based on the derivative of a difference of Gaussians [19]. All these solutions, which are based on the combination of smoothing and derivative filters, have the advantage of being robust to noise. Moreover, their expressions can be tuned according to the size of the edges involved. However they are not optimized for orientation estimation and do not guarantee a high precision estimation. The most critical cases occur on local maxima where the gradient is null and its orientation is undefined.

Different approaches, based on the use of a set of oriented filters, can also be found in literature. The filter shape depends on the type of structure to be identified: line, half-line, cross, "T-shape", etc. The orientation which maximizes the energy response of the filter is the one of the underlying texture pattern. The quadrature filters [20][21][22], the steerable filters [23][24] and the wedge steerable filters [25] fall into this category. These approaches provide with an estimation relying on a broader definition of the orientation not restricted to an edge but to any kind of structure. They are even able to detect various orientations occurring simultaneously on the same point. 
Recent works on the human visual system have shown the sensitivity of the visual cortex to specific frequencies and orientations in the case of 2-D-image perception as well as motion perception. Under this assumption, several authors (e.g. [26][1]) suggest that the local analysis of an image should be based on the local power spectrum density. The approximation of the spectrum by a set of Gabor filters [28] is a classical tool in computer vision but is not adapted to the accurate estimation of an orientation. On the contrary, the approach proposed by Bigün et al. [29] provide with a better accuracy. It consists in searching the axis of maximum inertia of the local power spectrum through a Principal Component Analysis.

Another family of solutions proposed in literature is based on the use of the covariance matrix of the local gradient field [19][10][18][11][30]. The formulations proposed by Kass and Witkin [19] and Rao [10][18][11] use complex representations of the gradients. Through a variance-based criterion minimization, they prove that the optimal estimation of the dominant orientation is given by the argument of the sum of the squared gradient vectors. Yet another formulation based on the Principal Component Analysis of the local Deriche-gradient field was proposed in [30]. All these techniques turn out to be equivalent to the approach based on the local Fourier transform [29]. Indeed, the square gradient formulation is simply a generalization of the double argument technique, which was first proposed by Mardia [31] and later by Baschelet [28], for statistics in biology. Naturally, these approaches make a statistical analysis of a set of gradient vectors. So, they are not adapted to our framework since we require that the orientation estimator remains local.

All these approaches provide with orientation estimations. However, most of the time they are not optimized for bias reduction and the attention devoted to noise robustness often leads to operators which are not local anymore and do not respect the constraint of scale.

This paper presents a new framework for the design of convolution masks dedicated to the specific problem of orientation estimation. In section 2, we will introduce a directional model for texture and give a general description of our convolution masks. In section 3, we will present the optimization scheme for bias reduction, address the issues of noise robustness and scale of analysis and finally give examples of unbiased orientation estimators. In section 4, we will exercise our operators on various directional textures, i.e. synthetic textures, seismic data, fingerprints and composite materials. We will also compare our operators to other efficient approaches, in terms of bias, noise immunity, and scale adaptation.

\section{The gradient and valleyness operators}

\subsection{The directional texture model}

In the following, we will assume that a directional texture can be locally modeled by a profile function $h$ which specifies the amplitude variation along a line orthogonal to the direction of the texture. Thus, for each pixel $(i, j)$, the intensity of an image $f$ is supposed to be defined by:

$$
f(i, j)=h(t),
$$

where $t=j \cos \theta-i \sin \theta$ and $\theta$ is the local texture orientation.

Figure 3 shows an example of such a texture with $h(t)=\sin (t)$ and $\theta=\pi / 8$.

This model is well fitted for directional textures with a unique and non ambiguous local orientation. It is similar to the linearly symmetric model proposed in [29]. In the rest of the paper we will use these definitions for directional textures and orientation. Despite the simplicity of this model, we will show that our approach remains relevant even on more complex texture models.

Let us now draw an analogy between image analysis and topography. The extrema of the profile function $h$ can be seen as crests and thalwegs, i.e. valley, of the topographic pattern. The maxima of the first derivative of $h$ form the lines of steepest slope. The main drawback of orientation estimation methods based on the gradient is their inadequacy on crests and valleys. In noisy cases, the correct orientation estimation is difficult near crest or valley lines where the gradient magnitude is very low. On the other hand, the gradient is perfectly adapted to the estimation of orientation on lines of steepest slope. These lines are located between crests and valley lines. On textures similar to the one of Figure 3 , such areas correspond to inflexion points. They will be called inflexion areas.

The approach we propose was previously briefly mentioned in [33][34]. It is based on the use of two complemen-

Fig. 4 tary operators. The first one is the classical gradient, which is adapted to high sloped regions. The second one will be called valleyness and is fitted to the crest and valley lines. Both operators are FIR filters. They are implemented using convolution masks that can be optimized for bias reduction and chosen to reduce the sensitivity to noise. In the following, we will present these operators in a general point of view and we will see how they can provide us with an orientation estimate. 


\subsection{The gradient operator}

The gradient approach is based on the convolution of the image with a horizontal mask $G_{x}$ and a vertical mask $G_{y}$. $G_{x}$ is anti-symmetric for the vertical axis and symmetric for the horizontal axis. $G_{y}$ derives from a $\pi / 2$ rotation of $G_{x}$. $G_{x}$ is composed of the set of coefficients defined by:

$$
G_{x}=\left(d_{i, j}^{g}\right)_{(i, j) \in S_{G}},
$$

where $S_{G} \subset \mathbb{Z}^{*} \times \mathbb{Z}$ is the support of the mask.

The symmetry properties involve that $\forall(i, j) \in S_{G}, d_{i, j}^{g}=d_{i,-j}^{g}=-d_{-i, j}^{g}$. In order to obtain FIR filters, $S_{G}$ is supposed

Fig. 5 bounded.

The gradient components $g_{x}$ and $g_{y}$ are computed by convolving the image with $G_{x}$ and $G_{y}$ :

$$
g_{x}=G_{x} * f, \quad g_{y}=G_{y} * f .
$$

The orientation of the pattern, which is orthogonal to the gradient vector, is then estimated at location $(i, j)$ by:

$$
\hat{\theta}(i, j)=\left\{\begin{array}{l}
\tan ^{-1} \frac{-g_{x}(i, j)}{g_{y}(i, j)}(\bmod \pi) \text { if } g_{y}(i, j) \neq 0 \\
\frac{\pi}{2} \text { otherwise }
\end{array},\right.
$$

where the function $\tan ^{-1}$ provides with an orientation belonging to $]-\frac{\pi}{2}, \frac{\pi}{2}[$.

\subsection{The valleyness operator}

\subsubsection{Principle}

The purpose of the valleyness operator is to perform an orientation estimation in cases where the gradient is inadequate i.e. near crests and valley lines. Thus, we have to define FIR filters the response of which is maximal on crests and valleys.

In the framework of texture inspection, Davies et al. [35] [36] have introduced the notion of line detector to detect the presence and estimate the orientation of patterns in a minimum number of operations. Their approach is based on the assumption that the grey level variation around a point located on a crest line is approximately sinusoidal.

Let define this variation $r$ by $r(\xi)=G \cos 2(\xi-\theta)$, where $\xi$ belongs to [0,2 $2 \pi$ and $\theta$ is the pattern orientation. $G$ is the magnitude of the variation at an arbitrary distance $\rho$ (Figure 6).

Fig. 6

Let now introduce the two integrals $v_{0}$ and $v_{\pi / 4}$ :

$$
v_{0}=\int_{0}^{2 \pi} p_{0}(\xi) r(\xi) d \xi \text { and } v_{\pi / 4}=\int_{0}^{2 \pi} p_{\pi / 4}(\xi) r(\xi) d \xi
$$

where $p_{0}(\xi)=\cos 2 \xi$ and $p_{\pi / 4}(\xi)=\sin 2 \xi$.

It can be shown that: $v_{0}=G \pi \cos 2 \theta$ and $v_{\pi / 4}=G \pi \sin 2 \theta$. Thus, we obtain the following estimation of $\theta \bmod \pi / 2$ :

$$
\theta=\frac{1}{2} \tan ^{-1} \frac{v_{\pi / 4}}{v_{0}}\left(\bmod \frac{\pi}{2}\right),
$$

where the $\pi / 2$ indeterminacy will be addressed in section 2.3.3.

Finally, the value of the orientation is available by computing the two integrals $v_{0}$ and $v_{\pi / 4}$. However, this result assumes that the intensity variation around a crest point is sinusoidal. Moreover, the function $r$ is not available in practice. The approach we follow is similar to Davis' one. It consists in replacing the calculation of the integrals $v_{0}$ and $v_{\pi / 4}$ by the convolution of the image with two FIR filters.

\subsubsection{Convolution masks and orientation estimation}

The generic shapes of the masks $V_{1}$ and $V_{2}$ used for convolution are given in Figure 7. They have two antisymmetry axis and two symmetry axis. The mask $V_{l}$ consists in the following set of coefficients:

$$
V_{1}=\left(d_{i, j}^{\nu 1}\right)_{(i, j) \in S_{V 1}} \text {, with } S_{V 1} \subset\left\{(i, j) \in \mathbb{Z}^{2} \mid i \neq j \text { and } i \neq-j\right\} \text {. }
$$

The symmetry properties involve that:

$$
\forall(i, j) \in S_{V 1}, d_{i, j}^{v 1}=d_{i,-j}^{v 1}=d_{-i, j}^{v 1} \text { and } d_{i, j}^{v 1}=-d_{j, i}^{v 1} .
$$


The diagonal mask $V_{2}$ consists in the set of coefficients defined by:

$$
V_{2}=\left(d_{i, j}^{v 2}\right)_{(i, j) \in S_{V 2}}, \text { where } S_{V 2} \subset \mathbb{Z}^{*} \times \mathbb{Z}^{*} .
$$

The symmetry properties for $V_{2}$ lead to the following relations:

$$
\forall(i, j) \in S_{V 2}, d_{i, j}^{v 2}=-d_{i,-j}^{v 2}=-d_{-i, j}^{v 2} \text { and } d_{i, j}^{v 2}=d_{j, i}^{v 2} .
$$

$S_{V 1}$ and $S_{V 2}$ are bounded so that the valleyness operator is implemented as a FIR filter. The valleyness components $v_{1}$ and $v_{2}$ are computed by convolving $V_{1}$ and $V_{2}$ with the image:

Fig. 7

$$
v_{1}=V_{1} * f, \quad v_{2}=V_{2} * f \text {. }
$$

The orientation of the pattern can be estimated using the valleyness components by the following equation:

$$
\hat{\theta}(i, j)=\frac{1}{2} \tan ^{-1} \frac{v_{2}(i, j)}{v_{1}(i, j)}\left(\bmod \frac{\pi}{2}\right) .
$$

\subsubsection{Orientation indeterminacy}

As assumed in section 2.1, the orientation of a pattern is defined modulo $\pi$. However, as the central pixel $(i, j)$ is not taken into account in the computation of $v_{1}$ and $v_{2}$, equation (12) entails an indeterminacy of $\pi / 2$ instead of $\pi$. Let us show this indeterminacy in a simple example. Figure 8 shows three $3 \times 3$ images. The three configurations only differ from the central pixel. In all cases, the computations of $v_{1}$ and $v_{2}$ will give the same results which reveal the $\pi / 2$ indeterminacy.

Fig. 8 In order to remove this difficulty, we propose to use the following orientation estimator:

$$
\hat{\theta}(i, j)=\frac{1}{2} \tan ^{-1} \frac{v_{2}(i, j)}{v_{1}(i, j)}+\varepsilon(i, j) \frac{\pi}{2}(\bmod \pi),
$$

where $\varepsilon(i, j)=\left\{\begin{array}{l}0 \text { if } f(i, j)>\bar{f}(i, j) \\ 1 \text { if } f(i, j)<\bar{f}(i, j)\end{array}\right.$.

$\bar{f}(i, j)$ denotes the mean grey level within a small neighbourhood around the current pixel $(i, j)$, for instance:

$$
\bar{f}(i, j)=\frac{1}{\operatorname{Card}\left(S_{V 1} \cup S_{V 2}\right)} \sum_{(k, l) \in S_{V 1} \cup S_{V 2}} f(i+k, j+l) \cdot
$$

By comparing the value of the central pixel with its neighbourhood, this formula gets rid of the indeterminacy in the case of simple patterns matching the directional texture model (see section 2.1). In the case of a perfect saddle configuration (Figure 8c), the orientation is indeterminate and should be weighted by a null confidence index considering that this configuration does not correspond to our textural model.

\section{Optimization of the operators}

In the previous section, we have introduced two generic operators, i.e. the gradient and the valleyness, which are likely to provide orientation estimates respectively near crests and valley lines. We will now propose an optimization scheme to reduce the bias of both operators. Indeed, as we will show it in section 4.1, the orientation estimated using classical gradient operators is biased. For instance, using Prewitt's operator, bias can reach $17^{\circ}$ for a texture with a sine profile. For many applications, i.e. anisotropic diffusion or texture characterization, such a bias is unacceptable. In the following we will also address the issue of noise sensitivity and we will provide examples of masks for the estimation of orientation at various scales.

\subsection{Bias reduction}

\subsubsection{Taylor series expansion of the image model}

Let consider the image as a sampling of a continuous and infinitely derivable function $f$ in the neighbourhood of a given pixel $\left(i_{0}, j_{0}\right)$. Thus the Taylor series expansion of $f$ gives:

$$
f\left(i_{0}+i, j_{0}+j\right)=\sum_{k=0}^{\infty} \frac{1}{k !}\left(i \frac{\partial}{\partial x}+j \frac{\partial}{\partial y}\right)^{k} f\left(i_{0}, j_{0}\right)=\sum_{k=0}^{\infty} \frac{1}{k !} \sum_{l=0}^{k}\left(\begin{array}{l}
k \\
l
\end{array}\right) i^{l} j^{k-l} \frac{\partial^{k}}{\partial x^{l} \partial y^{k-l}} f\left(i_{0}, j_{0}\right) .
$$

Using the directional texture model of section 2.1, the latter expression can be written as follows: 


$$
f\left(i_{0}+i, j_{0}+j\right)=\sum_{k=0}^{\infty} \frac{1}{k !} \sum_{l=0}^{k}\left(\begin{array}{l}
k \\
l
\end{array}\right) i^{l} j^{k-l} s^{l} c^{k-l}(-1)^{l} h^{(k)}\left(j_{0} c-i_{0} s\right)
$$

where $s$ and $c$ denote respectively the sine and the cosine of $\theta$.

\subsubsection{Unbiased gradient-based orientation estimation}

The gradient components $g_{x}$ and $g_{y}$, obtained by the convolution of $f$ with $G_{x}$ and $G_{y}$, can be written as follows:

$$
g_{x}\left(i_{0}, j_{0}\right)=\sum_{(i, j) \in S_{G}} d_{i, j}^{g} f\left(i_{0}+i, j_{0}+j\right) \quad \text { and } g_{y}\left(i_{0}, j_{0}\right)=\sum_{(i, j) \in S_{G}} d_{i, j}^{g} f\left(i_{0}+j, j_{0}+i\right) \text {. }
$$

Using the Taylor expansion of $f$ and the symmetry properties of the masks $G_{x}$ and $G_{y}$, these expressions can be developed as follows:

$$
\left\{\begin{array}{l}
g_{x}\left(i_{0}, j_{0}\right)=-4 \sum_{k \text { odd }} h^{k}\left(j_{0} c-i_{0} s\right) \sum_{\substack{l=1 \\
\text { odd }}}^{k} \frac{s^{l} c^{k-l}}{l !(k-l) !} \sum_{(i, j) \in S_{G}^{\prime}} i^{l} j^{k-l} a_{i, j} \\
g_{y}\left(i_{0}, j_{0}\right)=4 \sum_{k \text { odd }} h^{k}\left(j_{0} c-i_{0} s\right) \sum_{\substack{l=0 \\
\text { even }}}^{k-1} \frac{s^{l} c^{k-l}}{l !(k-l) !} \sum_{(i, j) \in S_{G}^{\prime}} i^{k-l} j^{l} a_{i, j}
\end{array}\right.
$$

where $S_{G}^{\prime} \subset \mathbb{N}^{*} \times \mathbb{N}$ is a reduced set of indices and the coefficients $a_{i j}$ are defined by:

$$
\left\{\begin{array}{l}
a_{i, j}=d_{i, j}^{g}, \quad \forall i \in \mathbb{N}^{*} \text { and } \forall j \neq 0, \\
a_{i 0}=\frac{1}{2} d_{i 0}^{g}, \forall i \in \mathbb{N}^{*} .
\end{array}\right.
$$

The expressions of $g_{x}$ and $g_{y}$ can then be used to obtain the optimization equation:

$$
\left\{\begin{array}{c}
K_{0}(\theta, i, j)=\frac{\sum_{k=0}^{+\infty} h^{(2 k+1)}\left(j_{0} c-i_{0} s\right) \sum_{i, j \in S_{G}^{\prime}} a_{i, j} A_{0, k}(i, j)}{\sum_{k=0}^{+\infty} h^{(2 k+1)}\left(j_{0} c-i_{0} s\right) \sum_{i, j \in S_{G}^{\prime}} a_{i, j} A_{1, k}(i, j)} \\
A_{0, k}(i, j)=\sum_{l=1}^{2 k+1} \frac{s^{l-1} c^{2 k+1-l}}{l !(2 k+1-l) !} i^{l} j^{2 k+1-l} \\
A_{1, k}(i, j)=\sum_{l=0}^{2 k+1} \frac{s^{2 k+1-l} c^{l-1}}{l !(2 k+1-l) !} i^{l} j^{2 k+1-l}
\end{array} .\right.
$$

The null bias condition for any profile function $h$ is $K_{0}\left(\theta, i_{0, j} j_{0}\right)=1$. This implies $A_{0, k}(i, j)=A_{l, k}(i, j)$ for any derivation order $k$. This is not possible with a FIR filter.

However, it is possible to get sub-optimal masks canceling the bias for a given order $n$ :

$$
\sum_{k=0}^{n} h^{(2 k+1)}\left(j_{0} c-i_{0} s\right) \sum_{i, j \in S_{G}^{\prime}} a_{i, j} A_{0, k}(i, j)=\sum_{k=0}^{n} h^{(2 k+1)}\left(j_{0} c-i_{0} s\right) \sum_{i, j \in S_{G}^{\prime}} a_{i, j} A_{1, k}(i, j) \text {. }
$$

The bias can then be canceled for image functions whose derivatives are null for orders larger than $2 n+1$ i.e. polynomial functions of degree $2 n+1$.

Indeed, the limitation of the Taylor expansion at order $n$ allows us to establish the null bias condition as the solution of a linear system of constraints which can be summarized by the following equation:

$$
\forall k<n, \sum_{i, j \in S_{G}^{\prime}} a_{i, j} A_{0, k}(i, j)=\sum_{i, j \in S_{G}^{\prime}} a_{i, j} A_{1, k}(i, j) .
$$

For derivation orders from 1 to 3 , this system is equivalent to the following unique constraint:

$$
\left(C_{1}^{G}\right) \quad 3 \sum_{i, j \in S_{G}^{\prime}} i j^{2} a_{i, j}=\sum_{i, j \in S_{G}^{\prime}} i^{3} a_{i, j} .
$$

For derivation orders 4 and 5, a second constraint appears: 


$$
\left(C_{2}^{G}\right) \quad 5 \sum_{i, j \in S_{G}^{\prime}} i j^{4} a_{i, j}=\sum_{i, j \in S_{G}^{\prime}} i^{5} a_{i, j} .
$$

Finally, for derivation orders 6 and 7, two more constraints must be fulfilled:

$$
\left(C_{3}^{G}\right) \sum_{i, j \in S_{G}^{\prime}} i^{7} a_{i, j}=6 \sum_{i, j \in S_{G}^{\prime}} i j^{6} a_{i, j} \text { and }\left(C_{4}^{G}\right) 5 \sum_{i, j \in S_{G}^{\prime}} i^{3} j^{4} a_{i, j}=3 \sum_{i, j \in S_{G}^{\prime}} i^{5} j^{2} a_{i, j} .
$$

Naturally, this approach can be extended to higher derivation order.

In order to reach high orders in our optimization scheme, we will need masks with a large number of coefficients, which implies increased mask sizes. We will see later in section 4.1 that the results obtained with small-sized masks are satisfactory and significantly better than the results obtained with other approaches[16][17][12].

\subsubsection{Unbiased valleyness-based orientation estimation} lows:

Using the procedure described in the previous section, the valleyness components $v_{1}$ and $v_{2}$ can be expressed as fol-

$$
\begin{aligned}
& v_{1}\left(i_{0}, j_{0}\right)=4 \sum_{\substack{k=0 \\
\text { even }}}^{\infty} h^{(k)}\left(j_{0} c-i_{0} s\right) \sum_{\substack{l=0 \\
\text { even }}}^{k} \frac{s^{l} c^{k-l}}{l !(k-l) !} \sum_{(i, j) \in S_{V 1}^{\prime}}\left(i^{l} j^{k-l}-j^{l} l^{k-l}\right) b_{i, j} \\
& v_{2}\left(i_{0}, j_{0}\right)=-4 \sum_{\substack{k \\
\text { even }}}^{\infty} h^{(k)}\left(j_{0} c-i_{0} s\right) \sum_{\substack{l=1 \\
\text { odd }}}^{k-1} \frac{s^{l} c^{k-l}}{l !(k-l) !} \sum_{(i, j) \in S_{V 2}^{\prime}}\left(i^{l} j^{k-l}+j^{l} i^{k-l}\right) c_{i, j}
\end{aligned}
$$

In these expressions, the reduced sets of coefficients $b_{i j}$ and $c_{i j}$ are defined by:

$$
\left\{\begin{array} { l } 
{ b _ { i , j } = d _ { i , j } ^ { v _ { 1 } } , \forall i \in \mathbb { N } ^ { * } , \forall j \neq 0 } \\
{ b _ { i , 0 } = \frac { 1 } { 2 } d _ { i , 0 } ^ { v _ { 1 } } , \forall i \in \mathbb { N } ^ { * } }
\end{array} \text { and } \left\{\begin{array}{l}
c_{i, j}=d_{i, j}^{v_{2}}, \forall i \in \mathbb{N}^{*}, i \neq j \\
c_{i, i}=\frac{1}{2} d_{i, i}^{v_{2}}, \forall i \in \mathbb{N}^{*}
\end{array} .\right.\right.
$$

The sets of indices $S_{V 1}^{\prime}$ and $S_{V 2}^{\prime}$ are depicted in Figure 7:

$$
S_{V 1}^{\prime} \subset\left\{(i, j) \in \mathbb{N}^{*} \times \mathbb{N}^{*} \mid j<i\right\} \quad \text { and } \quad S_{V 2}^{\prime} \subset\left\{(i, j) \in \mathbb{N}^{*} \times \mathbb{N}^{*} \mid j \leq i\right\} .
$$

As in section3.1.2, we can use the above Taylor expansions to derive the null bias constraints for the valleyness-based estimation. Taking into account these constraints, we provide with masks canceling the bias for derivation orders less than a chosen integer $2 n+1$.

From order 1 and 2, the unique constraint is:

$$
\left(C_{1}^{V}\right) \quad 2 \sum_{i, j \in S_{V 2}^{\prime}} i j c_{i, j}=\sum_{i, j \in S_{V 1}^{\prime}}\left(i^{2}-j^{2}\right) b_{i, j} .
$$

For orders 3 and 4, a second condition has to be fulfilled:

$$
\left(C_{2}^{V}\right) \quad 2 \sum_{i, j \in S_{V 2}^{\prime}} i j\left(i^{2}+j^{2}\right) c_{i, j}=\sum_{i, j \in S_{V 1}^{\prime}}\left(i^{4}-j^{4}\right) b_{i, j} .
$$

For orders 5 and 6, two new constraints appear:

$$
\left\{\begin{array}{l}
\left(C_{3}^{V}\right) 3 \sum_{i, j \in S_{V 2}^{\prime}} i j\left(i^{4}+j^{4}\right) c_{i, j}=\sum_{i, j \in S_{V 1}^{\prime}}\left(i^{6}-j^{6}\right) b_{i, j} \\
\left(C_{4}^{V}\right) \sum_{i, j \in S_{V 2}^{\prime}}\left(20 i^{3} j^{3}-6 i j\left(i^{4}+j^{4}\right)\right) c_{i, j}=\sum_{i, j \in S_{V 1}^{\prime}}\left(\left(i^{6}-j^{6}\right)-15 i^{2} l^{2}\left(i^{2}-j^{2}\right)\right) b_{i, j} .
\end{array}\right.
$$

At last, for order 7 and 8 , the new constraints are:

$$
\left\{\begin{array}{l}
\left(C_{5}^{V}\right) 4 \sum_{i, j \in S_{V 2}^{\prime}} i j\left(i^{6}+j^{6}\right) c_{i, j} d_{i, j}^{\nu_{2}}=\sum_{i, j \in S_{V 1}^{\prime}}\left(i^{8}-j^{8}\right) b_{i, j} \\
\left(C_{6}^{V}\right) 28 \sum_{i, j \in S_{V 2}^{\prime}}\left(i^{5} j^{3}+i^{3} j^{5}\right) c_{i, j}=\sum_{i, j \in S_{V 1}^{\prime}}\left(\left(i^{8}-j^{8}\right)-28 i^{2} l^{2}\left(i^{4}-j^{4}\right)\right) b_{i, j}
\end{array}\right.
$$

Like the gradient-based one, this approach can be extended to higher derivation order.

\subsection{Noise sensitivity reduction}

The cancellation of the bias for image functions of order $2 n+1$ requires to fulfill all the constraints $\left(C_{k}^{G}\right)$ and $\left(C_{k}^{V}\right)$ for $\mathrm{k} \leq \mathrm{n}$. For such a purpose, if we use masks with a number of available coefficients larger than $n$, the extra degrees 
of freedom provide us with subspaces on which the noise sensitivity reduction can be carried out. An optimization scheme was proposed in [33] in the case of a sinusoidal profile function. Besides, we have shown in [37] that the choice of the non-zero coefficients on the border of the mask reduces the noise sensitivity, whatever the profile function.

Once the location of the non-zero coefficients is set, fulfilling the sets of constraints $\left(C_{k}^{G}\right)$ and $\left(C_{k}{ }^{V}\right)$ allows us to obtain unbiased convolution masks.

\subsection{Optimized mask design}

Taking into account the sections 3.1 and 3.2, we propose the following framework for the design of robust gradient and valleyness masks:

Choose the order of the Taylor expansion. This choice entails the number of coefficients and the size of the masks.

Choose the location of the non-zero coefficients according to the noise sensitivity considerations.

Solve the $\left(C_{k}^{G}\right)$ and $\left(C_{k}{ }^{V}\right)$ systems which give the mask coefficients.

In Figure 9, we provide with examples of convolution masks for the horizontal component of the Gradient-based OPerator, for various sizes (GOP3, GOP5, GOP7, GOP9). The corresponding horizontal components are obtained by a $\pi / 2$ rotation of these masks.

Fig. 9 GOP3 is unbiased for polynomial profile functions $h$ of order less or equal to 3 . GOP5 is unbiased up to order 5. GOP7 and GOP9 are unbiased up to the order 7.

In Figure 10, we provide with the convolution masks for both components of the Valleyness-based OPerator,

Fig. 10 VOP3, VOP6 and VOP8.

VOP3 is unbiased for polynomial profile functions of order not greater than 2. VOP6 and VOP8 are unbiased up to order 6 .

\section{Results and discussion}

\subsection{Bias evaluation}

Thanks to our framework, GOP and VOP operators give an unbiased estimation of the orientation for any polynomial profile function so long as its order is appropriate. Nevertheless, it appears that for natural texture such as those in Figure 1, sine profile functions are more relevant than polynomials. Thus, let us consider the profile function: $h(u)=\sin (2 \pi u / T)$, where $T$ stands for the texture period. Unfortunately, $h(u)$ is infinitely derivable, so we cannot give a strictly unbiased orientation estimation with FIR filters.

Fig. 11 It can be shown [37] that the orientation bias obtained with both gradient and valleyness-based operators is uniform all over the texture. This bias only depends on the orientation $\theta$ and the period $T$ of the profile function. For any gradient-based operator, the orientation tangent estimate can be expressed as follows:

$$
\tan \hat{\theta}\left(i_{0}, j_{0}\right)=\frac{\sum_{i, j \in S_{G}^{\prime}} a_{i, j} \sin (\omega i \sin \theta) \cos (\omega j \cos \theta)}{\sum_{i, j \in S_{G}^{\prime}} a_{i, j} \sin (\omega i \cos \theta) \cos (\omega j \sin \theta)}, \forall\left(i_{0}, j_{0}\right) \in \mathbb{Z}^{2}, \text { with } \omega=\frac{2 \pi}{T} .
$$

The absolute estimation bias $|\hat{\theta}-\theta|$ can be compared with biases obtained using operators of similar sizes. Figure 11 shows the bias versus the period $T$ for a sinusoidal texture. The orientation chosen for this experiment is $\theta=\pi / 8$ which represents the worst case from the bias point of view, for all gradient operators [37]. GOP3 and GOP4 are less biased than Prewitt, Sobel and Deriche $(\alpha=2)$.

In the case of the Valleyness operator, the estimation of the orientation tangent is given by the following relationship.

$$
\tan \hat{\theta}\left(i_{0}, j_{0}\right)=\frac{\sum_{i, j \in S_{V 2}^{\prime}} c_{i, j}(\sin (\omega i \cos \theta \sin (\omega j \sin \theta+\sin (\omega i \sin \theta \sin (\omega j \cos \theta)))))}{\sum_{i, j \in S_{V 1}^{\prime}} b_{i, j}(\cos (\omega i \sin \theta \cos (\omega j \cos \theta-\cos (\omega i \cos \theta \cos (\omega j \sin \theta)))))}, \forall\left(i_{0}, j_{0}\right) \in \mathbb{Z}^{2} \text {, with } \omega=\frac{2 \pi}{T} .
$$

Figure 12 shows the bias of the valleyness-based orientation estimate in the case of a sinusoidal profile function. The orientation chosen for this experiment is the one which represents the worst case from the bias point of view. The

Fig. 12 
results for VOP6 are compared with the orientation obtained from Deriche's optimal second order derivative $(\alpha=2)$ [17]. VOP6 show the lowest bias for any texture period.

It should be noted that some very specific texture periods could be critical for GOP or VOP masks. For example, when the size of a vertical GOP mask is equal to the vertical period of the texture (see Figure 13), the convolution of the mask with the profile function gives a null response which induces an erroneous orientation estimation. Of course, similar problems occur with valleyness masks.

Fig. 13 In order to avoid this drawback, a sufficient solution consists in choosing the mask size smaller than the smallest period appearing in the texture.

\subsection{GOP and VOP combination, confidence and scale adaptability}

The gradient and valleyness operators can be used separately. However, the combination of these two operators can provide a more accurate orientation estimate in any pixel whether it is on an extremum area, on an inflexion area or anywhere between. Such a combination could be achieved in linear and non linear ways.

In the present paper we choose a non linear combination. It consists in selecting the most appropriate operator for each pixel depending on the local configuration (see Figure 14). On characteristic pixels (i.e. crest/valley or inflexion areas) we choose either the valleyness or the gradient-based operators with a suitable scale. On intermediate areas, we use a combination of the orientations computed on neighbouring characteristic pixels.

Fig. 14

The different steps for the computation of the complete orientation map are the following:

- detection of characteristic pixels,

- selection of the operator scales according to the distance between neighbouring inflexion or crest/valley areas,

- computation of the orientations on characteristic pixels with the appropriate operators,

- computation of the orientations on intermediate areas.

The whole approach will be denoted GV-JOE (Gradient and Valleyness based Joint Orientation Estimation). Let us detail the steps of the approach.

\subsubsection{Characteristic pixels}

The gradient operator provides optimal orientation estimations on inflexion areas and the valleyness operator provides optimal orientation estimations on crest/valley areas.

On any crest/valley pixel, the local scale is derived from the distance between the crest/valley area to which it belongs and the nearest other crest/valley area. The local orientation is then computed by the valleyness operator with the appropriate size.

On any inflexion pixel, the local scale is derived from the distance between the inflexion area to which it belongs and the nearest crest/valley area. The local orientation is then computed by the Gradient operator with the appropriate size.

Note that the size of the masks could be fixed according to a pre-processing stage or to the a priori known period of the underlying texture [33].

\subsubsection{Deriving confidence values}

It is convenient to associate each orientation estimate with a measure of its relevance [29]. Such a measure will be called a confidence index. Usually, confidence indexes are based on the modulus of the filter response [26][18]. In our case, the modulus resulting from the convolution of our masks is not suitable. Indeed, as we combine various masks in the same image, the corresponding response modulus can not be compared.

Therefore, we compute the same confidence index $\eta$ for all pixels whether they are processed by a gradient-based or a valleyness-based operator. We choose the confidence of the line operator which has been introduced in [37]. This index is based on the computation of the grey level variance along the estimated direction of the texture. The more the texture is oriented, the smaller is the variance and the bigger is the coherence.

We will also use this confidence index in the next step to compute the orientation for non characteristic pixels.

\subsubsection{Intermediate areas}

At this point, orientations $\theta$ and confidence values $\eta$ are available only on characteristic pixels (inflexion and crest/valley areas). Orientations of intermediate pixels are not known: they are assigned null confidence values.

Orientations and confidences can be obtained on intermediate pixels by combining the orientations and confidences of neighbouring characteristic pixels. This is carried out by implementing a smoothing filter. As the distance to the nearest characteristic pixel is not known a priori, an infinite impulse response (IIR) filter is more appropriate. To re- 
duce computational time, we choose Deriche's 2D smoothing filter [17] which allows a recursive implementation. The coefficients of this filter are given by:

$$
w(k, l)=(1+\alpha|k|)(1+\alpha|l|) e^{-\alpha(|k|+|l|)}
$$

The lower the parameter $\alpha$ is, the faster the coefficients $w(k, l)$ decrease. To adapt the filter to the most critical cases (i.e. high spatial frequencies), the value of parameter $\alpha$ must be high. It appears experimentally that the choice of $\alpha=1.5$ is appropriate for most applications, especially when spatial periods are in the range [2, 20] pixels. Nevertheless, when spatial periods exceed 20 pixels, an $\alpha$ value taken in $[0.5,1.5]$ could be more appropriate.

In this filtering stage, orientations are handled as directional data [31].

In previous works [34][37], we proposed a filling process based on a Fast Marching algorithm [39].

\subsection{Noise robustness}

In order to evaluate the noise immunity of GV-JOE approach, we compute a Mean Angular Deviation $(M A D)$ indicator for various synthetic textures:

$$
M A D=\frac{1}{N} \sum_{\text {pixels }(i, j)} \Delta(\hat{\theta}(i, j), \theta(i, j)),
$$

where $N$ is the size of the sample (i.e. the number of pixels $(i, j)$ considered), $\theta$ stands for the theoretical orientation and $\Delta\left(\theta_{1}, \theta_{2}\right)=\min \left(\left|\theta_{1}-\theta_{2}\right|, \pi-\left|\theta_{1}-\theta_{2}\right|\right)$.

The $M A D$ indicator takes into account both the bias and the noise sensitivity, i.e. variance.

The GV-JOE approach is compared to other orientation estimators with equivalent scales. We compute a Principal Component Analysis (PCA) of the local Deriche gradient field with parameter $\alpha=1.0$ [30]. We also show the result of the wedge steerable filters [25], with size $15 \times 15$, constructed from 18 interpolation filters, and the steerable filters E2, with size $13 \times 13$ [23]. As a matter of information, we have also reported the results obtained with Prewitt gradient and with Deriche gradient, with parameter $\alpha=1.0$, without PCA.

Let us note that for each competing approach, we tuned the parameters in order to achieve the best orientation estimation, keeping the computing support size comparable. Of course, in the case of texture showing a unique orientation (Figure 3), better estimations could be obtained increasing the size of steerable or wedge steerable filters or decreasing parameter $\alpha$ of Deriche's gradient. However, the scale of the operators would not be comparable anymore. Furthermore, in order to obtain a local estimation, it is essential to keep the size of the operator as small as possible when the orientation varies inside the image (Figure 16).

Figure 15 displays the $M A D$ indicator versus $\theta$ for the sinusoidal texture introduced in section 4.1 ( $T_{0}=4$ pixels). The sample images have been corrupted by a white noise with an $\mathrm{SNR}=1 \mathrm{~dB}$. The GV-JOE provides the lowest $M A D$

Fig. 15 values. Moreover these values remain almost constant whatever the orientation whereas the other operators, except the wedge and steerable filters, are not isotropic. As the wedge filters are asymmetric, they use only half of the computing support. This can explain the poor results obtained with these filters.

It should be noted that for larger values of $T_{0}$ and SNR, e.g. $T_{0}=10$ and SNR=10dB, steerable filters E2, PCA on

Fig. 16 Deriche gradient field and GV-JOE give similar MAD values. Additional results are available in [37].

The GV-JOE approach has also been exercised on a non-stationary rippled texture, in order to show its relevance for local orientation estimation. Figure 16 provides an example of such a picture.

Figure 17 shows the behaviour of the MAD indicator versus the SNR for the most reliable orientation operators,

Fig. 17 considering the previous experiment. On such textures, the GV-JOE combination gives the most accurate estimation for SNR up to $5 \mathrm{~dB}$. For higher SNR values, GV-JOE results are close to the steerable filter ones.

\subsection{Application to a natural texture}

We now apply our operators to the fingerprint picture in Figure 18a. The color-palette used for orientation is given in Figure 18e.

We compute the most efficient operators regarding to the $M A D$ indicator to the fingerprint image (a): Steerable Fil-

Fig. 18 ters $(13 * 13)(\mathrm{b})$, PCA on Deriche gradient Field (c) and finally our GV-JOE combination (d). Figure 18 (d) exhibits a more regularized orientation field. Moreover, the GV-JOE combination highlights sharply the singular points of the fingerprint. Thus GV-JOE appears to be both selective and robust to noise.

The GV-JOE operator has also been successfully exercised on a large set of both synthetic and real textures. Detailed results can be found in [37].

\section{Conclusion}


In this paper we have proposed a new approach for orientation estimation. This approach hinges on two classes of convolutive operators based on the gradient and valleyness estimation. We have provided a framework for their optimization regarding bias reduction and noise robustness.

The two classes of operators have been combined in order to address three major objectives. First, the complementarity of these operators allows us to ensure the estimation accuracy at each location. Indeed our GV-JOE combination consists in applying the operators where they are the most accurate, i.e. the gradient on inflexion pixels, the valleyness on crests and valleys. Elsewhere, a combination of neighboring inflexion, crest and valley orientations is computed. Secondly, in order to respect the variation of the texture scale in the image, we have implemented an adaptive selection of the size of our operators. Thirdly, we associate a confidence index with each orientation estimate. For such a purpose we compute the line operator which is based on the grey level variance along the estimated direction of the texture.

We have exercised our approach both on synthetic and natural textures. These experiments have shown that, when used separately, both classes of operators are more accurate than classical derivative approaches. In noisy cases, the GV-JOE implementation has improved the robustness of our operators. Moreover, compared to well known orientation estimators, it gives the best estimates in the most difficult cases i.e. for high frequency textures and low SNR.

Work is in progress to first derive new texture characterization and segmentation approaches and secondly, extend them to 3-D orientation estimation in various fields as seismic data processing or medical imaging.

\section{References}

[1] B. Julesz, J.R. Bergen, Textons, the fundamental elements in preattentive vision and perception of textures, The Bell System Technical Journal, vol.62, no. 6, (1983), pp. 1619-1645.

[2] A.R. Rao, G.L. Lohse, Identifying high level features of texture perception, CVGIP: Graphical Models and Image Processing, vol. 55 , no. 3, (1993), pp. 218-233.

[3] A. Jain, I. Hong, R. Bolle, On-line fingerprint verification, IEEE Trans. on PAMI, vol. 19, no. 4, (1997), pp. $302-313$.

[4] A. Ross, A. Jain, J. Reisman, A hybrid fingerprint matcher, Pattern Recognition, vol. 36, no. 7, (2003), pp. 1661-1673.

[5] J.P. Da Costa, C. Germain, P. Baylou, A curvilinear approach for textural feature extraction, in Proc. of Quality Control and Artificial Vision, Le Creusot, France, (2001), pp. 541-545.

[6] C. Germain, J.P. Da Costa, O. Lavialle, P. Baylou, Multiscale estimation of vector field anisotropy application to texture characterization, Signal Processing, vol. 83, issue 7, , (2003) pp. 1487-1503.

[7] V.A. Kovalev, M. Petrou, Y.S. Bondar, Using orientation tokens for object recognition, Pattern Recognition Letters, vol. 19, no. 12, (1998), pp. 1125-1132.

[8] C. Germain, J.P. Da Costa, P. Baylou, Multiscale estimation of textural features. Application to the characterization of texture anisotropy, in: Proceedings of ICPR, Barcelona, Spain, (2000), pp. 935-938.

[9] R. Azencott, C. Graffigne, C. Labourdette, Edge detection and segmentation of textured plane images, in: Stochastic models, statistical method in image analysis, P. Baronne, A. Frigessi, M. Piccioni Editors, Springer Verlag, (1990), pp. 75-88.

[10] A.R. Rao, A taxonomy for texture description and identification, Springer Verlag, New York, (1990).

[11] A.R. Rao, R.C. Jain, Computerized flow field analysis: oriented texture fields, IEEE Transactions on PAMI, vol. 14, no 7 , (1992), pp. 693-709.

[12] E.P. Lyvers, O.R. Mitchell, Precision edge contrast and orientation estimation, IEEE Transactions on PAMI, vol. 10, no 6, (1988), pp. 927-937.

[13] V. Kovalev, M. Petrou, Multidimensional co-occurrence matrices for object recognition and matching, Graphical Models and Image Processing, vol. 58, no. 3, (1996), pp. 187-197.

[14] P.E. Danielsson, O. Seger, Rotation invariance and higher order derivative detectors, Computer Vision Graphics and Image Processing, vol. 49, (1990), pp. 198-221.

[15] J.F. Canny, A computational approach to edge detection, IEEE Trans. on PAMI, vol. 8, no. 6, (1986), pp. 679-698.

[16] R. Deriche, Using Canny's criteria to derive a recursively implemented optimal edge detector, International Journal of Computer Vision, vol. 1, no. 2, (1987), pp. 167-187.

[17] R. Deriche, Fast algorithms for low-level vision, IEEE Transactions on PAMI, vol. 12, no. 1, (1990), pp. 78-87.

[18] A.R. Rao, B.G. Schunck, Computing oriented texture fields, CVGIP: Graphical Models and Image Processing, vol. 53, no. 2, (1991).

[19] M. Kass, A. Witkin, Analysing oriented patterns, Computer Vision Graphics Image Processing, vol. 37, 1987, pp.362-385.

[20] P. Perona, J. Malik, Detecting and localizing edges composed of steps, peaks and roofs, in: Proc. of the Int. Conf. on Computer Vision, (1990), pp.52-57.

[21] H. Knutsson, M. Andersson, Robust n-dimensional orientation estimation using quadrature filters and tensor whitening, in: Proceedings of ICASSP, (1994).

[22] M. Anderson, H. Knutsson, Orientation estimation in ambiguous neighbourhoods, in: Proceedings of SCIA, (1991).

[23] W.T. Freeman, E.H. Adelson, The design and use of steerable filters, IEEE Trans. on PAMI, 13(9), (1991), pp. 891-906.

[24] P. Perona, Deformable kernels for early vision, IEEE Trans. on PAMI, vol. 17, no. 5, (1995), pp. 488-499.

[25] E.P. Simonselli, F. Hany, Steerable wedge filters for local orientation analysis, IEEE Trans. on Image Processing, vol. 5, no. 9, (1996), pp. 1377-1382.

[26] G. H. Granlund, In search of a general picture processing operator, Computer Graphic Image Processing, vol. 8, (1978), pp. 155-173. 
[27] E.H. Adelson, J.R. Bergen, Spatiotemporal energy models for the perception of motion, Journal of the Optical Society of America, vol. 1, no. 2, (1985), pp. 284-299.

[28] D. Gabor, Theory of Communication, JIEE, no 26, (1946), pp. 429-459.

[29] J. Bigün, G.H. Granlund, J. Wiklund; Multidimensional orientation estimation with applications to texture analysis and optical flow, IEEE Trans. on PAMI, vol. 13, no. 8, (1991), pp. 775-789.

[30] M. Donias, Caractérisation de champs d'orientation par analyse en composantes principales et estimation de la courbure. Application aux images sismiques, PhD Thesis, University Bordeaux 1, (1999), in French.

[31] K.V. Mardia, Statistics of directional data, Academic Press, London and New York, (1972).

[32] E.B. Batschelet, Circular statistics in biology, Academic Press, New York, (1981).

[33] J.P. Da Costa, F. Le Pouliquen, C. Germain, P. Baylou, New Operators for Optimized Orientation Estimation, in: Proceedings of ICIP'01, Thessaloniki, Greece, (2001).

[34] F. Le Pouliquen, C. Germain, P. Baylou, Scale adaptive orientation operator, in: Proceedings of ICPR, Montreal, Canada, (2002).

[35] E. R. Davies, D. R. Mason, M. Bateman, J. Chambers, C. Ridgway, Linear feature detectors and their application to cereal inspection, in: Proc. of EUSIPCO, pp. 2561-2564, Rhodes, Greece, (1998).

[36] E.R. Davies, Some problems in food and cereal inspection and methods for their solution, in: Proc. of Quality Control and Artificial Vision, pp. 35-46, Le Creusot, France, (2001).

[37] F. Le Pouliquen, Opérateurs discrets pour l'estimation adaptative et optimale de l'orientation. Application à l'imagerie sismique. PhD Thesis, University Bordeaux 1, France, (2003), in French.

[38] F. Le Pouliquen, C. Germain, P. Baylou, Line orientation operator, in: Proceedings of ICIP, Thessaloniki, Greece, (2001).

[39] J.A. Sethian, Level Set Methods : Evolving Interfaces in Geometry, Fluid Mechanics, Computer Vision and Material Sciences, Cambridge University Press, Cambridge, UK, (1996). 


\section{Vitae:}

Franck LE POULIQUEN received the Diploma of Electronic Engineering from the ENSEIRB (French National Graduate School of Electronic Engineering of Bordeaux) in 1997, and a PhD degree in Image Processing at the University of Bordeaux in 2003. His main research interest is textural image analysis, particularly orientation, and fault recognition in textured pictures. Applications concern seismic data analysis.

Jean-Pierre DA COSTA received the Diploma of Electronic Engineering from the ENSEIRB (French National Graduate School of Electronic Engineering of Bordeaux), in 1998 and a PhD degree in Image Processing at the University of Bordeaux in 2001. He is currently Assistant Professor at ENITA of Bordeaux. His main research interest is textural image analysis and particularly orientation, statistics of orientation fields, anisotropy and structural pattern recognition. Applications concern composite material and high resolution remote sensing.

Christian GERMAIN received a BSc degree in computer science in 1984 from Amiens University and a PhD degree from the University of Bordeaux in 1997. He has been teaching computer science and image processing since 1984 and he is currently Assistant Professor at ENITA of Bordeaux. He is carrying out research at the University of Bordeaux I in the Signal an Image Processing team. His research interests include textural picture analysis and processing, Markov random fields for segmentation, and he is presently focused on anisotropy, curvature and orientation in 2-D and 3-D pictures. Applications concern composite material, high resolution remote sensing and seismic data.

Pierre BAYLOU received his Engineer Diploma from the Electronic Engineering School of Bordeaux (ENSEIRB) in 1964 and his PhD degree in communication circuits from the University of Bordeaux in 1968. He has been teaching electronics, computer science and image processing at the ENSEIRB since 1966. From 1980 to 1990, he developed autonomous robots devoted to open field agriculture at the LARFRA automatics laboratory. In 1990, he joined the Signal and Image Team where he is now in charge of a research group in the image analysis and understanding field. His research interests include adaptive filtering, texture analysis, deformable models and adaptive mathematical morphology. The main application fields are seismic data understanding and composite material image analysis. 

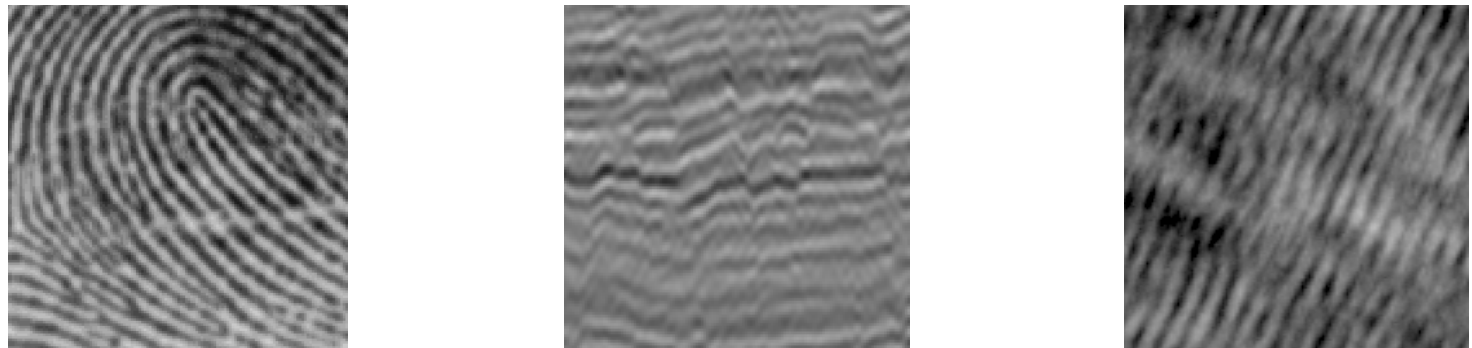

Figure 1. Examples of directional textures: fingerprint (left), seismic layers (middle), atomic structure in a composite material image (right). 


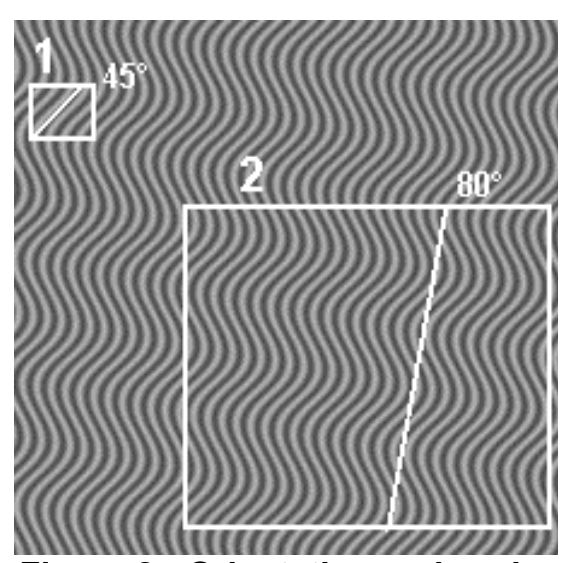

Figure 2. Orientation and scale:

1. local orientation is $45^{\circ}$

2. large scale orientation is $80^{\circ}$ 


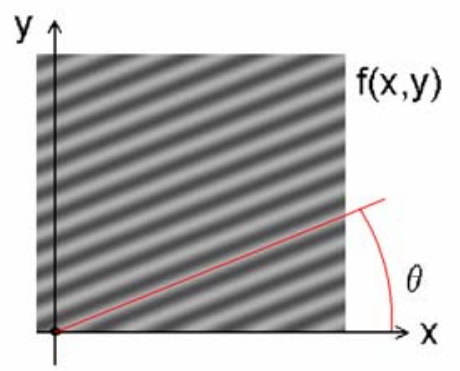

Figure 3. Example of directional texture:

$f(x, y)$ : grey level 2-D function

$\theta$ : texture orientation 


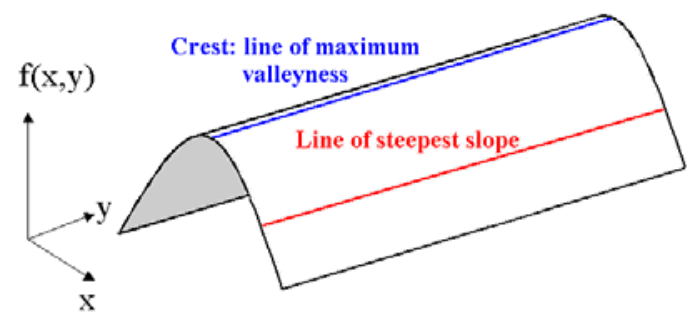

Figure 4. Steepest slope and crest lines on the topographic view of a directional texture 


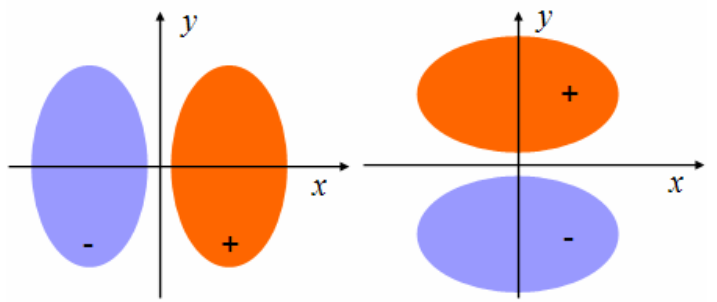

Figure 5. Generic shapes of the Gradient masks: horizontal and vertical symmetry of the masks 

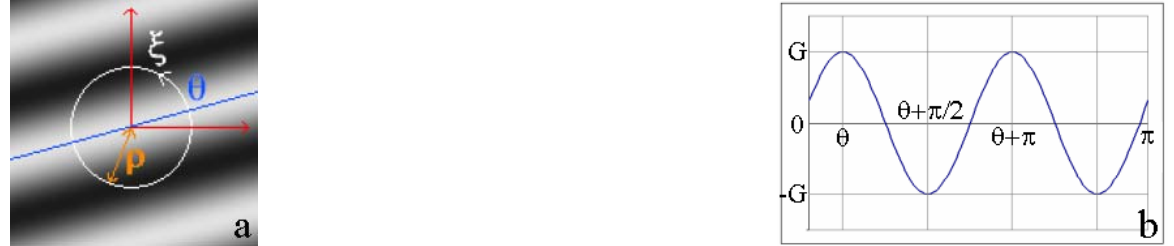

Figure 6. Valleyness principle: (a) sinusoidal texture; (b) intensity variation around the crest point at a distance $\rho$. 

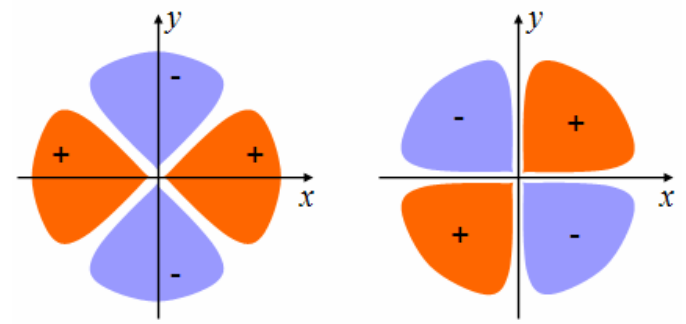

Figure 7. Generic shapes of the Valleyness masks: central symmetry of the masks 

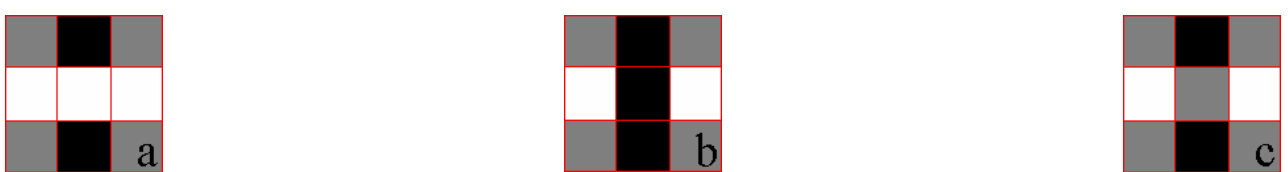

Figure 8. $\pi / 2$ indeterminacy in a simple case: (a) the orientation is the one of the white crest; (b) the orientation is the one of the black line; (c) the orientation is indeterminate. 


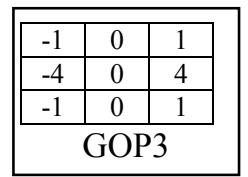

\begin{tabular}{||c|c|c|c|c|}
\hline-7 & 0 & 0 & 0 & 7 \\
\hline-32 & 0 & 0 & 0 & 32 \\
\hline-12 & 0 & 0 & 0 & 12 \\
\hline-32 & 0 & 0 & 0 & 32 \\
\hline-7 & 0 & 0 & 0 & 7 \\
\hline \multicolumn{5}{|c|}{ GOP5 } \\
\hline
\end{tabular}

\begin{tabular}{|c|c|c|c|c|c|c|}
\hline-41 & 0 & 0 & 0 & 0 & 0 & 41 \\
\hline-216 & 0 & 0 & 0 & 0 & 0 & 216 \\
\hline-27 & 0 & 0 & 0 & 0 & 0 & 27 \\
\hline-272 & 0 & 0 & 0 & 0 & 0 & 272 \\
\hline-27 & 0 & 0 & 0 & 0 & 0 & 27 \\
\hline-216 & 0 & 0 & 0 & 0 & 0 & 216 \\
\hline-41 & 0 & 0 & 0 & 0 & 0 & 41 \\
\hline \multicolumn{7}{|c|}{ GOP7 } \\
\hline
\end{tabular}

\begin{tabular}{|c|c|c|c|c|}
\hline 0 & 0 & 0 & 0 & 0 \\
\hline 0 & -6 & 0 & 6 & 0 \\
\hline-1 & -16 & 0 & 16 & 1 \\
\hline 0 & -6 & 0 & 6 & 0 \\
\hline 0 & 0 & 0 & 0 & 0 \\
\hline \multicolumn{5}{|c|}{ GOP4 } \\
\hline
\end{tabular}

\begin{tabular}{||c|c|c|c|c|c|c|c|c|}
\hline-7377 & 0 & 0 & 0 & 0 & 0 & 0 & 0 & 7377 \\
\hline-37584 & 0 & 0 & 0 & 0 & 0 & 0 & 0 & 37584 \\
\hline-6216 & 0 & 0 & 0 & 0 & 0 & 0 & 0 & 6216 \\
\hline-48048 & 0 & 0 & 0 & 0 & 0 & 0 & 0 & 48048 \\
\hline 0 & 0 & 0 & 0 & 0 & 0 & 0 & 0 & 0 \\
\hline-48048 & 0 & 0 & 0 & 0 & 0 & 0 & 0 & 48048 \\
\hline-6216 & 0 & 0 & 0 & 0 & 0 & 0 & 0 & 6216 \\
\hline-37584 & 0 & 0 & 0 & 0 & 0 & 0 & 0 & 37584 \\
\hline-7377 & 0 & 0 & 0 & 0 & 0 & 0 & 0 & 7377 \\
\hline
\end{tabular}

Figure 9. Example of GOP masks at scales 3, 5, 7 and 9. 


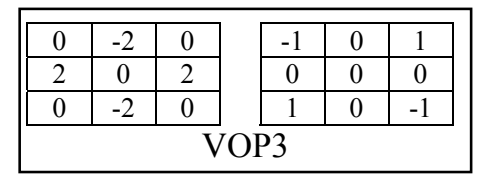

\begin{tabular}{|c|c|c|c|c|c|c|}
\hline 0 & 0 & 0 & -116 & 0 & 0 & 0 \\
\hline 0 & 0 & -426 & -1890 & -426 & 0 & 0 \\
\hline 0 & 426 & 0 & 0 & 0 & 426 & 0 \\
\hline 116 & 1890 & 0 & 0 & 0 & 1890 & 116 \\
\hline 0 & 426 & 0 & 0 & 0 & 426 & 0 \\
\hline 0 & 0 & -426 & -1890 & -426 & 0 & 0 \\
\hline 0 & 0 & 0 & -116 & 0 & 0 & 0 \\
\hline
\end{tabular}

\begin{tabular}{|c|c|c|c|c|}
\hline 0 & -1254 & 0 & 1254 & 0 \\
\hline-1254 & -564 & 0 & 564 & 1254 \\
\hline 0 & 0 & 0 & 0 & 0 \\
\hline 1254 & 564 & 0 & -564 & -1254 \\
\hline 0 & 1254 & 0 & -1254 & 0 \\
\hline
\end{tabular}

\section{VOP6}

\begin{tabular}{|c|c|c|c|c|c|c|c|c|c|c|c|c|c|c|c|}
\hline 0 & 0 & 0 & 0 & -12 & 0 & 0 & 0 & 0 & & & & & & & \\
\hline 0 & 0 & 0 & -33 & 0 & -33 & 0 & 0 & 0 & 0 & -16 & -28 & 0 & 28 & 16 & 0 \\
\hline 0 & 0 & 0 & 0 & 0 & 0 & 0 & 0 & 0 & -16 & 0 & 0 & 0 & 0 & 0 & 16 \\
\hline 0 & 33 & 0 & 0 & 0 & 0 & 0 & 33 & 0 & -28 & 0 & 0 & 0 & 0 & 0 & 28 \\
\hline 12 & 0 & 0 & 0 & 0 & 0 & 0 & 0 & 12 & 0 & 0 & 0 & 0 & 0 & 0 & 0 \\
\hline 0 & 33 & 0 & 0 & 0 & 0 & 0 & 33 & 0 & 28 & 0 & 0 & 0 & 0 & 0 & -28 \\
\hline 0 & 0 & 0 & 0 & 0 & 0 & 0 & 0 & 0 & 16 & 0 & 0 & 0 & 0 & 0 & -16 \\
\hline 0 & 0 & 0 & -33 & 0 & -33 & 0 & 0 & 0 & 0 & 16 & 28 & 0 & -28 & -16 & 0 \\
\hline 0 & 0 & 0 & 0 & -12 & 0 & 0 & 0 & 0 & & & & & & & \\
\hline
\end{tabular}

Figure 10. Example of VOP masks at scales 3,6 and 8. 


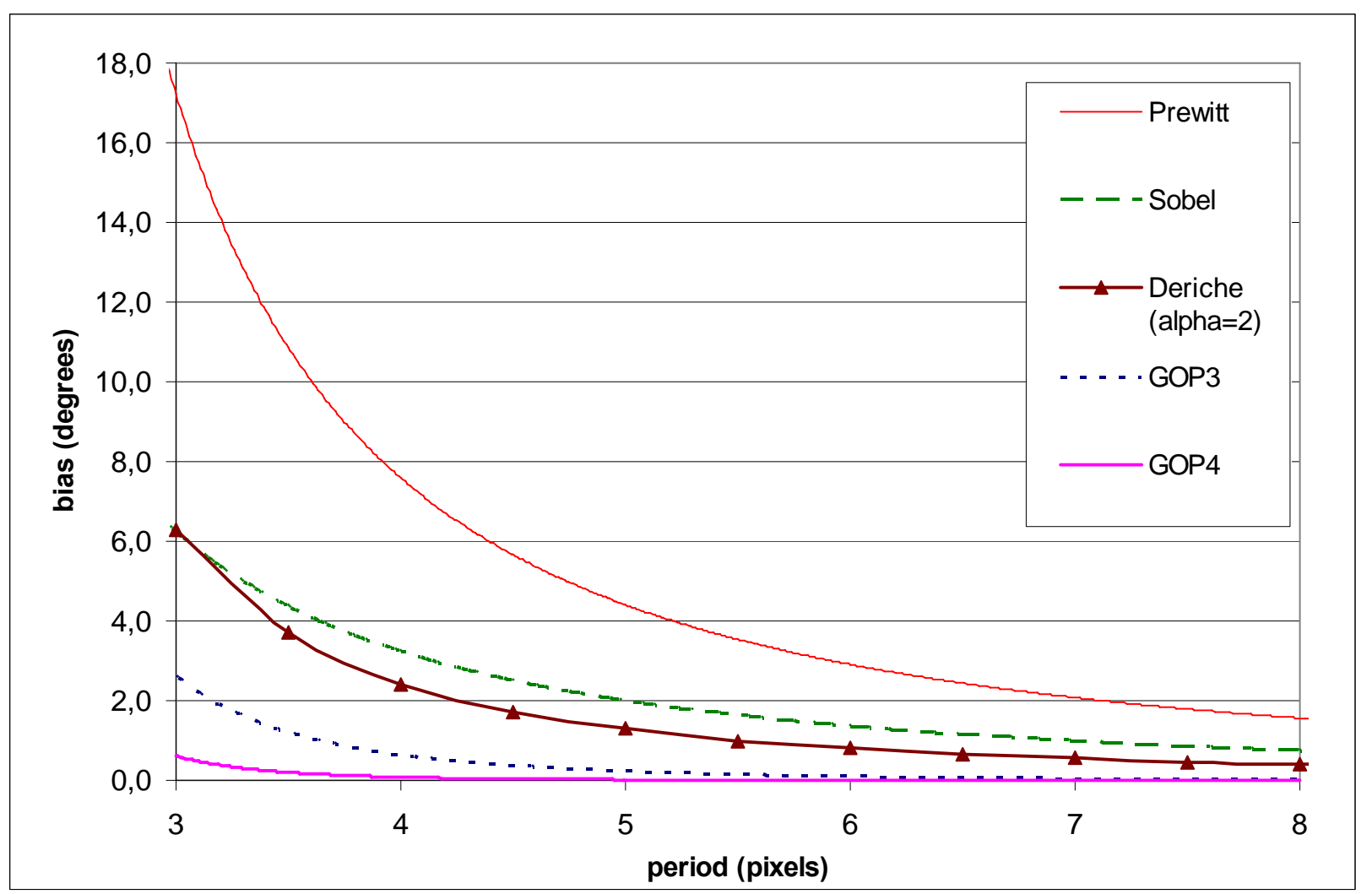

Figure 11. Maximum orientation bias for Gradient operators versus the period of the sine profile function (no noise). 


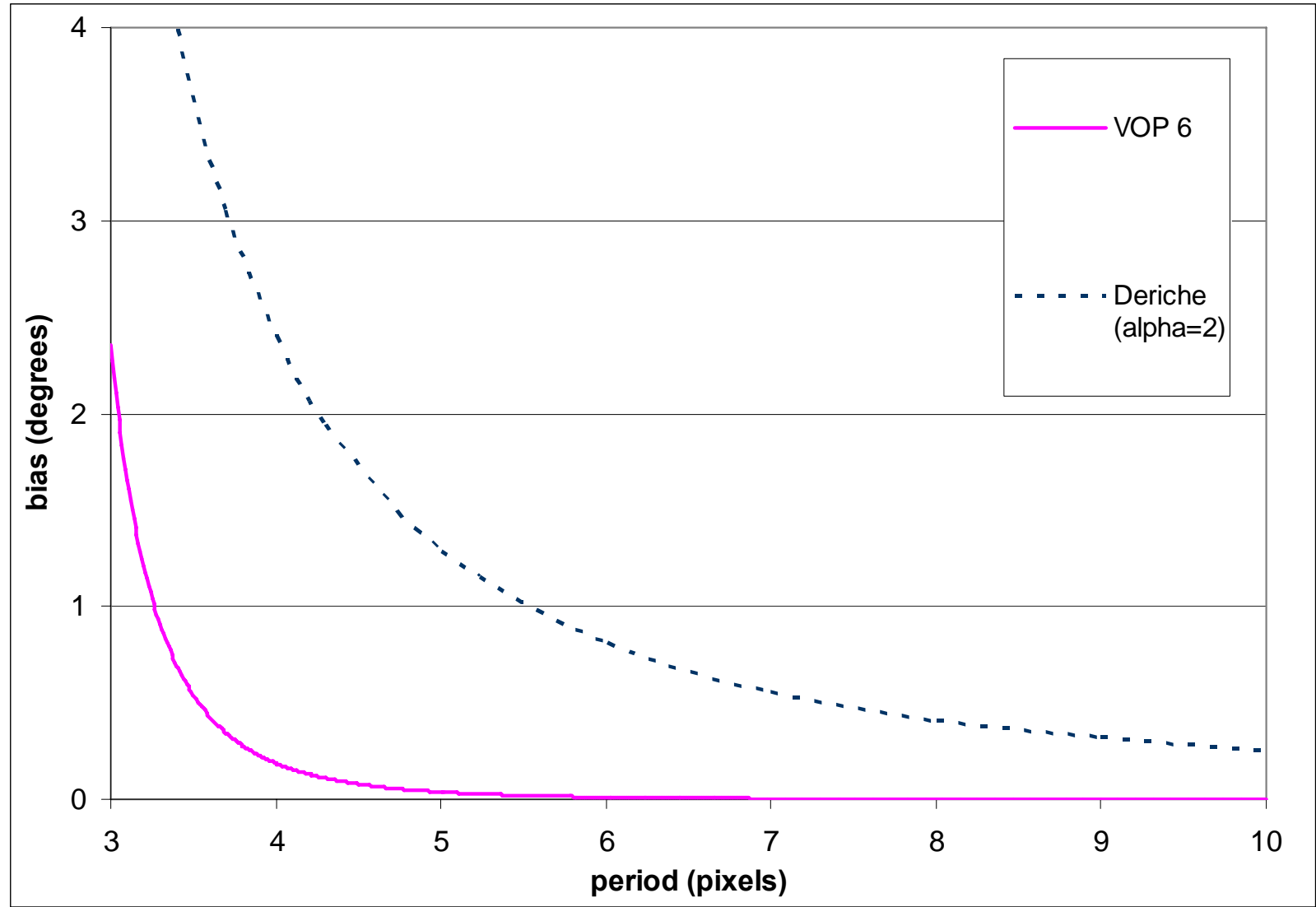

Figure 12. Maximum orientation bias for Valleyness operators versus the period of the sine profile function (no noise). 


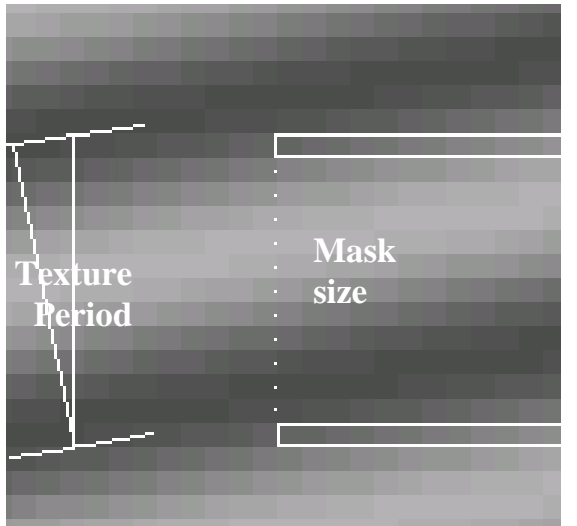

Figure 13. Critical configuration for a vertical gradient mask: the mask size is equal to the vertical period of the texture 


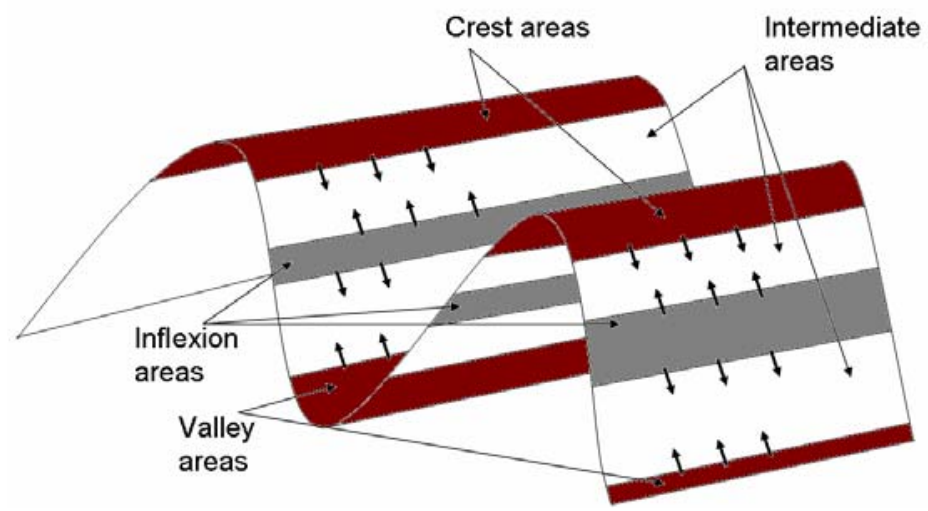

Figure 14. Principle of the GV-JOE operator

GOP filters are applied within inflexion areas VOP filters are applied within crests and valleys GOP and VOP values are propagated inside intermediate areas 


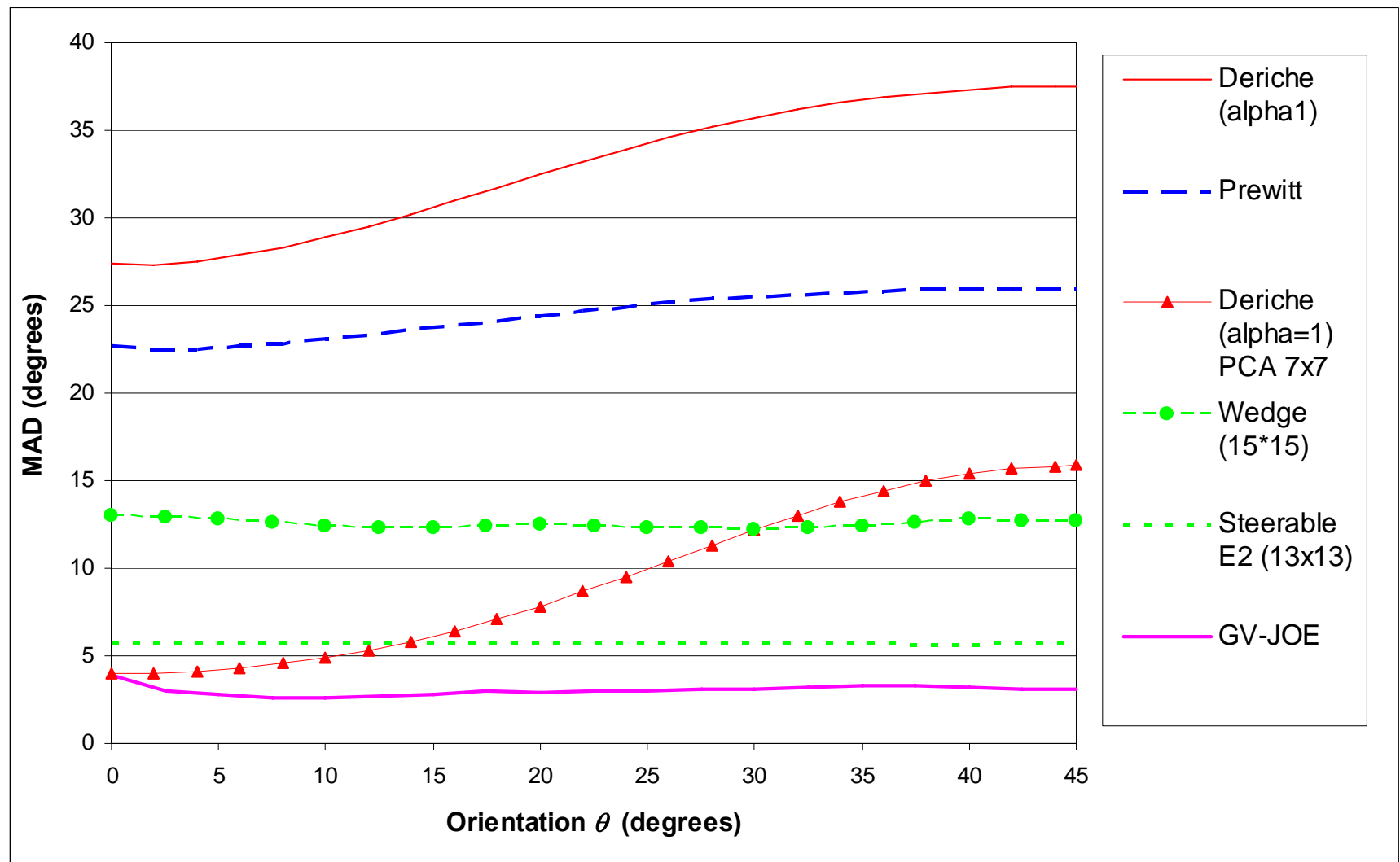

Figure 15. Mean Angular Deviation (MAD) versus theoretical orientation $\theta$ for sinusoidal textures $\left(T_{0}=4\right.$ pixels, $\left.S N R=1 \mathrm{~dB}\right)$. 


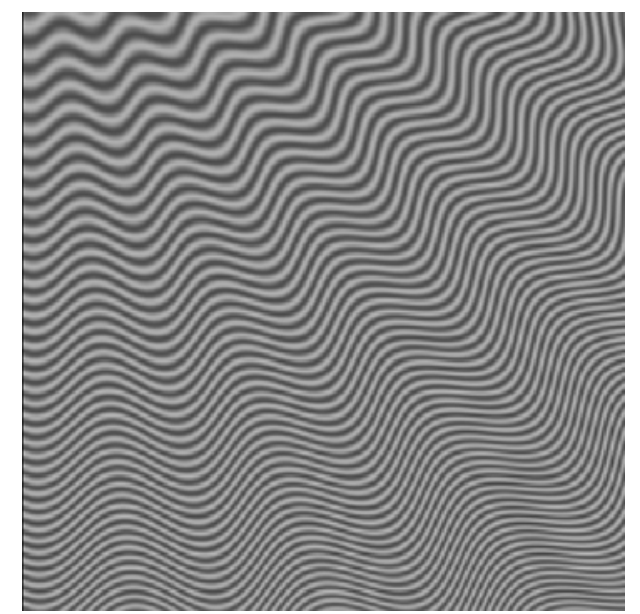

Figure 16. Concentric lines modulated in orientation and frequency. 


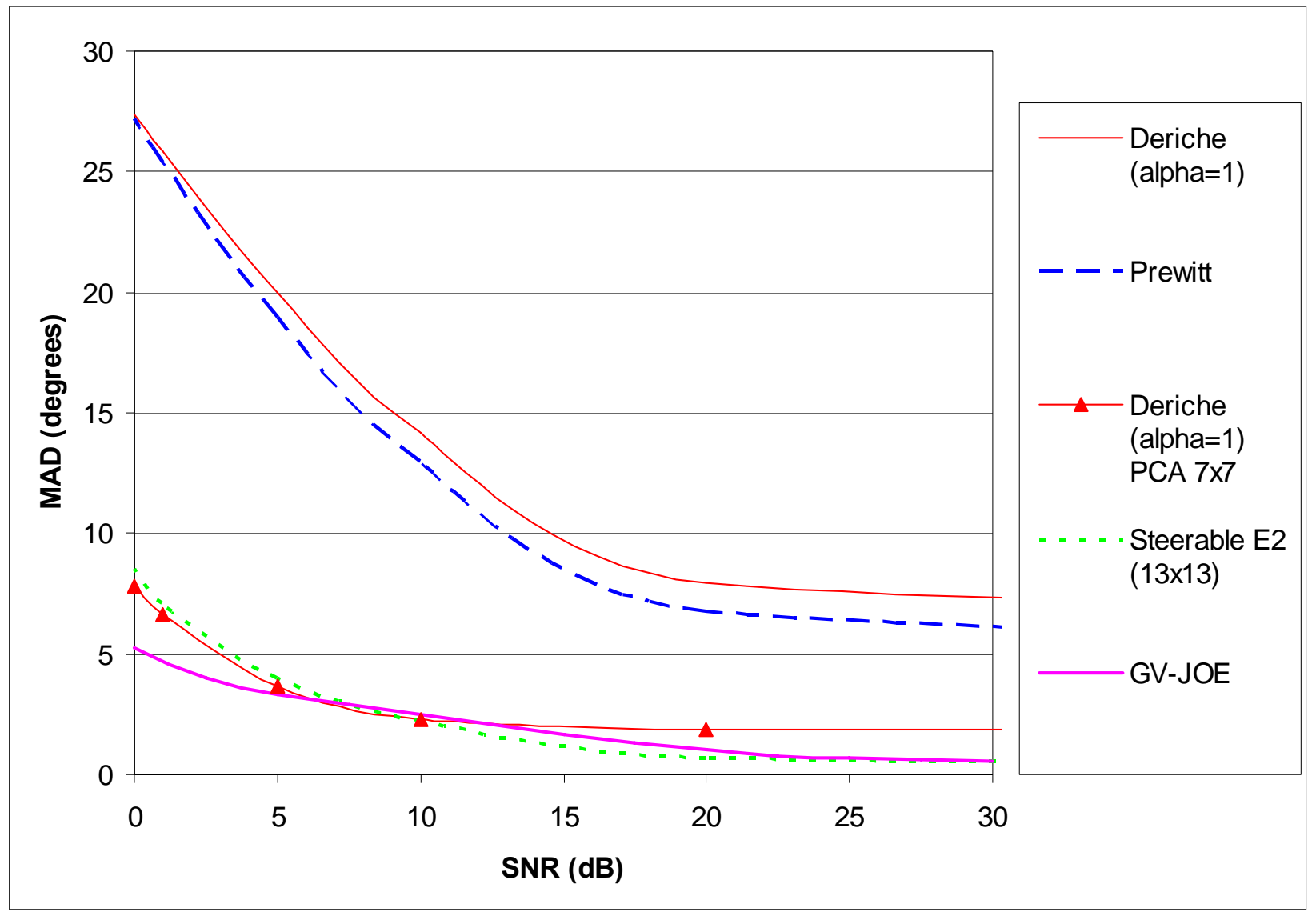

Figure 17. Mean Angular Deviation (MAD) versus SNR for concentric lines modulated in orientation and frequency. 


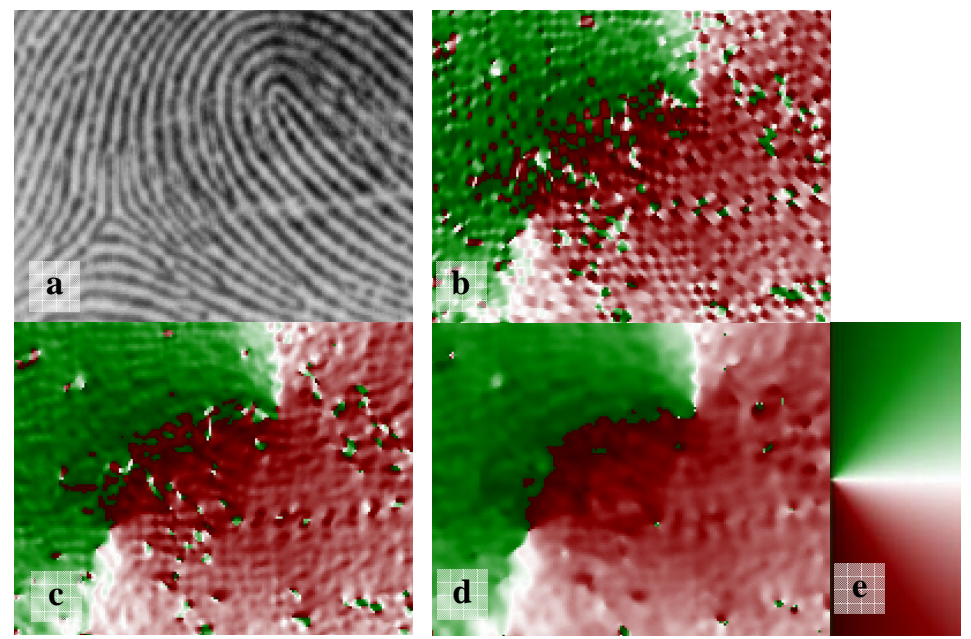

Figure 18. Orientation on a fingerprint image: (a) Fingerprint image.

(b) orientation with 13x13 E2 steerable filters; (c) orientation using PCA on Deriche's gradient field (with $\alpha=1.0$ ); (d) orientation using GV-JOE operator; (e) Colour palette for orientation 


\section{Illustration captions:}

1. Examples of directional textures: fingerprint (left), seismic layers (middle), atomic structure in a composite material image (right)

2. Orientation and scale.

3. Example of directional texture.

4. Steepest slope and crest lines on the topographic view of a directional texture.

5. Generic shapes of the Gradient masks.

6. Valleyness principle: (a) sinusoidal texture; (b) intensity variation around the crest point at a distance $\rho$.

7. Generic shapes of the Valleyness masks.

8. $\pi / 2$ indeterminacy in a simple case: (a) the orientation is the one of the white crest; (b) the orientation is the one of the black line; (c) the orientation is indeterminate.

9. Example of GOP masks at scales 3, 5, 7 and 9.

10. Example of VOP masks at scales 3, 6 and 8.

11. Maximum orientation bias for Gradient operators versus the period of the sine profile function (no noise)

12. Maximum orientation bias for Valleyness operators versus the period of the sine profile function (no noise).

13. Critical configuration for a vertical gradient mask.

14. Principle of the GV-JOE operator.

15. Mean Angular Deviation $(M A D)$ versus theoretical orientation $\theta$ for sinusoidal textures $\left(T_{0}=4\right.$ pixels, $\mathrm{SNR}=1 \mathrm{~dB}$ ).

16. Concentric lines modulated in orientation and frequency.

17. Mean Angular Deviation $(M A D)$ versus SNR for concentric lines modulated in orientation and frequency.

18. Orientation on a fingerprint image. 